\title{
Investigation of Fatigue Crack Models by Micro-scale Measurement of Crack Tip Deformation
}

\author{
D. Nowell ${ }^{1}$, K.I. Dragnevski ${ }^{2}$, and S.J. O'Connor ${ }^{2}$ \\ ${ }^{1}$ Department of Mechanical Engineering, Imperial College London, Exhibition Road, \\ London SW7 2AZ, UK \\ ${ }^{2}$ Department of Engineering Science, University of Oxford, Parks Road, Oxford OX1 3PJ, UK \\ d.nowell@imperial.ac.uk
}

\section{Abstract}

The paper describes displacement measurements taken in the vicinity of a fatigue crack tip using digital image correlation. In-situ loading is employed in a scanning electron microscope to get measurements very close to the tip. The results are compared to the usual elastic model of crack tip deformation and to the Christopher, James and Patterson model, which is critically discussed. It is shown that the use of an elastic model with a residual stress intensity caused by crack tip shielding gives a good representation of the experimental results.

\section{Introduction}

Engineering systems need to operate safely under a wide range of loads, which are often cyclic in nature. The two main approaches to fatigue life prediction are 'safe life', where the predicted life is based on experiments conducted to failure and a resulting S- $\mathrm{N}$ ' curve, and 'damage tolerance', which relies solely on crack propagation data. The latter approach has a number of advantages, and is therefore becoming increasingly popular. The principal advantage stems from the observation that crack propagation rates show less 'scatter' than total lives, because of the relative independence from microstructure. Hence an estimate of propagation life between the largest flaw to remain undetected in an NDT examination and failure can be reasonably reliable. Hence, one can achieve a positive assurance of safety, together with economic management of component life. The main disadvantage of the damage tolerant approach is the cost of regular NDT examinations, but these are steadily becoming more automated and less expensive.

Most damage tolerant life prediction methods employ the Paris 'Law' [1] which gives a power law relationship between the crack growth rate, $d a / d N$, and the range of stress intensity factor, $\Delta K$. This 'law' is justifiably popular, but one should remember that it is simply an empirical curve fit to laboratory data obtained at constant remote load amplitude. Real structures can show quite different load histories. For example, aircraft wings will be subject to varying load caused by turbulence, and offshore structures will see wave loading which may be almost random in nature. Hence, recent research has acknowledged that simple elastic crack models may have limited applicability, particularly when there is significant plastic deformation ahead of the crack and a corresponding plastic wake. However, development of improved models, such as those suggested by Pommier and Hammam [2], or Christopher et al. [3] requires good quality experimental data from propagating fatigue cracks.

A number of techniques have been employed to measure crack tip strains or displacements, either in two or three dimensions. In 2004, Fellows and Nowell [4] employed moiré interferometry to measure crack tip closure. This produced good results, but the experimental technique was difficult and time 
consuming. The availability of low cost digital cameras, combined with commercial or open-source software, has made Digital Image Correlation a popular means of measuring displacements in experiments [5]. Some early experiments were carried out by de Matos [6], but a number of researchers have since carried out similar measurements, including Yoneyama et al [7], Roux et al [8], and Vasco-Olmo [9]. Most experiments concentrate on evaluating the stress intensity factors for a conventional elastic approach. However, recently, Bahrli et al. [10] have used DIC data combined with finite elements to calculate J-Integrals for a propagating fatigue crack.

Measuring the displacements on the surface of a specimen is less demanding than techniques which acquire full 3D data through the specimen thickness. However, the 3D techniques provide useful information about the transition from 'plane stress-like' conditions at the surface to plane strain towards the centerline. Maire and Withers provide an overview of quantitative X-Ray tomography [11], and the application of the technique to fatigue cracks has been investigated by Limodun and coworkers [12], amongst others. An alternative to tomography is to use synchrotron X-Rays to investigate the elastic strain field around a fatigue crack tip. Some recent work in this area has been reported by Lopez-Crespo et al [13].

Our own work at Oxford was presented at the Forni di Sopra [14], Malaga [15], and Urbino [16] IJ Fatigue/FFEMS workshops. We have used DIC at macro- and micro- scales to determine near tip displacements fields. We have concentrated particularly on measuring the crack opening as a function of position along the line of the crack, since this quantity relatively easy to determine. If a direct measure of displacement, such as DIC, is used in the experiments, then this location is particularly attractive since the crack flanks are the largest displacements available, once rigid body motions are eliminated. The simplest model to use in interpreting the experimental results is the familiar elastic solution, first proposed by Westergaard [17]. Within the Westergaard framework of a sharp crack tip in an elastic material, the dominant term in the series expansion of stresses and strains is $1 / V_{r}$, where $r$ is the distance from the crack tip. Hence, the leading term in the expansion for displacements is $\sqrt{ }$. It is therefore possible to plot the relative displacement between the crack faces as a function of $V_{r}$ and to obtain the stress intensity factor for the crack. Experimental values may not correspond to those obtained from standard elastic solutions, since the latter do not account for crack tip shielding effects due to plasticity ahead of the crack tip, together with the plastic wake.

Because of the limitations involved in applying the elastic Westergaard equations to a fatigue crack with a plastic process zone and a plastic wake, a number of models have been proposed in recent years to account for plasticity effects whilst still retaining a largely elastic model. One of the simplest of these is that proposed by Pommier and Hammam [2]. In their approach the displacement field around the crack is modelled as a superposition of a conventional Westergaard elastic field, with a stress intensity factor $\mathrm{K}$, and a plastic field, which may be determined by finding the difference between an elastic-plastic FE analysis of the crack and a purely elastic one. This second field is also scaled by an 'intensity factor', $\rho$, although of course in this case no singularity is involved. Our own earlier work [15] has attempted to apply this approach to experimental results, but the modification to the elastic field was found to be relatively modest, and somewhat dependent on the assumed position for the crack tip in the images which are analysed. Our conclusion, therefore, was that the additional complexity involved in the model may not be justified in many practical cases. Typical results from the purely elastic approach are shown in Figures 1 and 2. In Figure 1 we show the variation of crack opening with $V_{r}$, for different levels of applied loading. It may be seen that the data 
form almost straight lines, meaning that the normal elastic $\mathrm{K}$-solution is a good fit to the experimental results at this length scale.

In Figure 2 we plot the variation of experimental stress intensity factor with applied load, derived from the slope of lines such as those shown in Figure 1. It is apparent from this figure that the stress intensity factor derived from the experiment is lower than that predicted by elastic theory. Over a significant proportion of the loading history the difference between the two lines is almost constant and this may be attributed to a negative residual stress intensity factor caused by the residual stress field surrounding the crack. Of course, the concept of a negative stress intensity factor does not make sense on its own, since compressive loading does not cause a singularity at the crack tip. However, a negative stress intensity factor can be a useful concept when combined with a positive $\mathrm{K}$ caused by loading, such that the overall stress intensity factor is positive. Hence, the experimental values of $K$ fall to zero once the applied stress intensity factor is less than the magnitude of the residual one. This forms a quantitative means of assessing the concept of crack tip shielding.

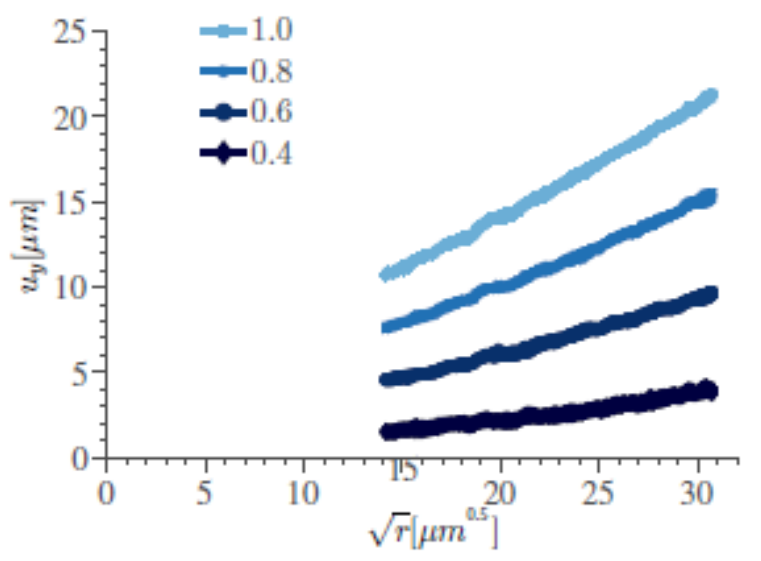

Figure 1: Variation of distance between pairs of points on either side of the crack with $\sqrt{ } \mathrm{r}$, where $r$ is the distance from the crack tip. The lines are for different values of $P / P_{\max }$

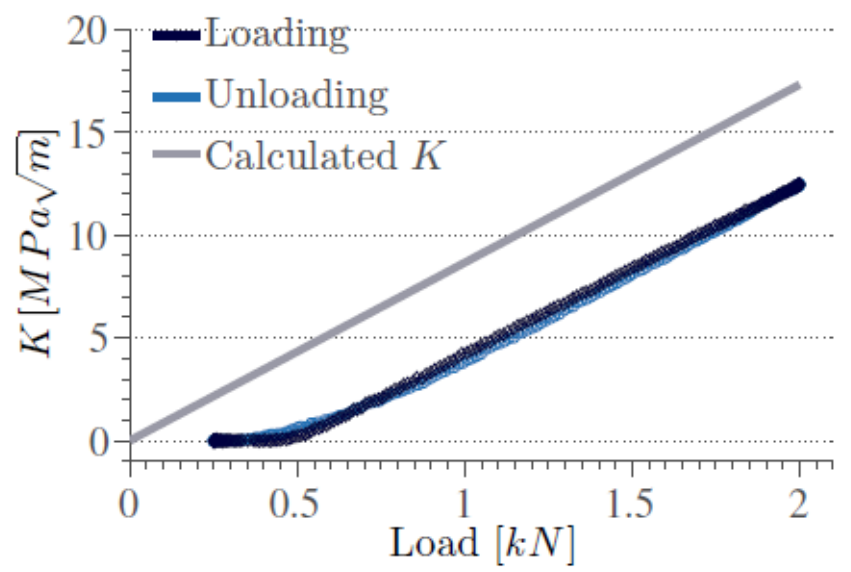

Figure 2: Variation of measured and calculated stress intensity factor with load, for a typical DIC experiment with a constant amplitude load history [14] 
A second model which has been proposed in recent years is that by Christopher, James, and Patterson, (often referred to as the CJP model) [3]. The CJP model attempts to capture some of the additional phenomena generated by a fatigue crack with a plastic wake. This approach leads to a crack description with four parameters as follows:

$\mathrm{K}_{\mathrm{F}} \quad$ The 'Forward Stress Intensity Factor', which is essentially similar to the applied $\mathrm{K}_{\mathrm{I}}$ in a conventional analysis

$\mathrm{K}_{\mathrm{R}} \quad \mathrm{A}$ 'Retardation Stress Intensity Factor', which arises as a result of the residual stress field set up by the wake, and which might be thought similar to the offset shown in Figure 2.

$\mathrm{K}_{\mathrm{S}} \quad \mathrm{A}$ 'Shear Stress Intensity' describing the shear present between the plastic wake and the surrounding elastic material

$\mathrm{T} \quad$ The conventional T-stress or bounded term in the Williams expansion.

It is easy to misunderstand some of these terms, so a brief further explanation will be given here with the aid of a diagram (Fig. 3) modified from that presented by James et al. in [3]. First, it is essential to understand that the plastic zone at the tip of the crack creates a wake along the crack faces, as the crack propagates, Hence, there is a plastic enclave of the approximate shape shown in grey in Fig. 3. If one then considers the boundary between this enclave of plastically deformed material and the surrounding elastic hinterland there may be $x, y$ and shear forces transmitted across this interface. In each case, the force on the enclave by the elastic hinterland will be equal and opposite to that exerted by the enclave on the elastic material. Figure 3 shows the forces exerted on the plastic enclave.

\section{Forces on plastic field}

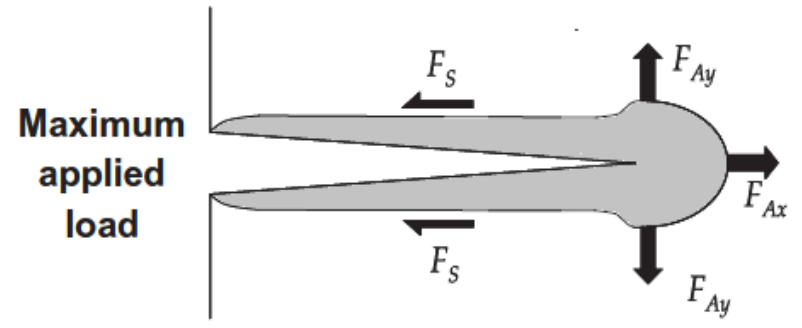

(a)

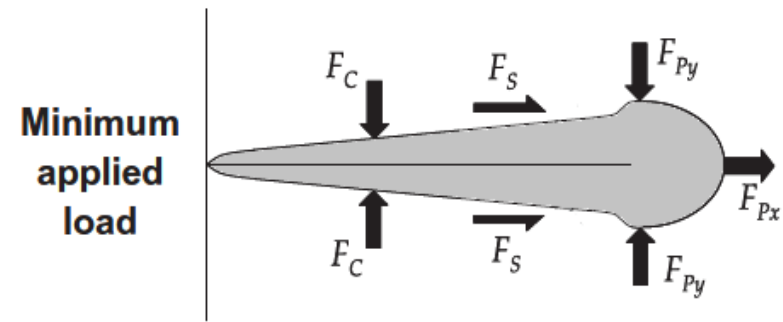

(b)

Figure 3: Forces between the plastic enclave (grey), and the surrounding elastic material (after [3]).

Starting with Fig. 3a, at maximum load there is an applied force opening the crack and causing tensile yield. This is labelled $F_{A y}$ in the figure. Similarly, there may be shear at both top and bottom of the enclave, and these forces (which for mode I must be in the same direction) are labelled $F_{s}$ in Fig.3a. Since the enclave must be in equilibrium, these two shear components must be reacted by a horizontal force, termed $\mathrm{F}_{\mathrm{Ax}}$. The authors of [3] include a separate term for the T-stress, but this is clearly not a net force across the interface, rather it is a stress component which occurs at the crack tip. Hence, it is perhaps more helpful to think of the T-stress as being a bounded effect caused by $F_{A x}$, which clearly does not cause any stress intensity at the crack tip. At the minimum applied load, James 
et al. [3] consider the crack at least partially closed (Fig. 3b.). They then show $F_{S}$ reversed in sign, which seems plausible, since the crack has reached this state by unloading from the maximum load. The $F_{x}$ force however, is not reversed in sign, so that if Fig. $3 b$ is considered as a free body diagram, then the plastic enclave would not be in equilibrium. What James et al do, however, change is the notation for the $x$-direction force, calling it now $F_{P_{x}}$ presumably to show that the plastic zone is somehow the entity responsible for the force, rather than the applied load. Of course, in the general case, both applied load, and plastic zone resistance contribute to the force across the interface, and in our view it is not possible (or perhaps helpful) to separate them. In the vertical direction, the sign of the main vertical force is reversed, and the notation is changed. Further, an additional force $F_{C}$ is introduced to represent the contribution to vertical force caused by crack closure. James et al. [3], then go on to collapse the plastic enclave onto the line of the crack, and develop a Muskhelishvili stress function for the surrounding elastic material. This eventually results in the four terms defined above.

Our own work has so far focused on simpler one or two parameter models, and our view is that the introduction of different forces with different notations and signs in Fig. 3., whilst intended to link the model to physical phenomena is not,in the end, particularly helpful. What is helpful, however, is the concept of a plastic wake and its effect on the surrounding material. A simpler approach would therefore acknowledge that there is no need to introduce different y-direction forces at minimum load. One can simply stick with the system of forces shown in Fig. 3a and drop the double subscripts. So, $F_{y}$ causes crack opening and a stress intensity, $F_{s}$, exerts shear on the areas above and below the crack, and this is reacted by $F_{x}$, which may be thought responsible for the bounded stress component in the $x$-direction (T-stress). These three forces neatly map onto the four terms tabulated on the previous page:

$\mathrm{F}_{\mathrm{S}} \quad$ Is responsible for $\mathrm{K}_{\mathrm{S}}$. Physically, its link to crack propagation rates is difficult to see, so that one might consider it, at most a secondary effect.

$F_{x} \quad$ Is responsible for the T-stress. Again, this is acknowledged to be a secondary effect, though perhaps more important than $\mathrm{K}_{\mathrm{S}}$.

$\mathrm{F}_{\mathrm{y}} \quad$ Must clearly be responsible for $\mathrm{K}_{\mathrm{F}}$ and $\mathrm{K}_{\mathrm{R}}$, and which may be separated using the CJP approach, or if preferred may remain as a single $K_{I}$ term.

\section{Macro-scale experimental results}

Before applying the approach described above to micro scale experimental results, it is helpful to apply the analysis to some previous work published in the literature. We have essentially extracted the dominant $K_{1}$ term in the above simplified version of the CJP model, and an example result has already been presented in Fig. 2. To compare this with the results of the full model, we will use some results produced by Vasco Olmo [9]. He used a full-field DIC technique to extract $K_{F}$ and $K_{R}$ for the CJP model, using essentially the same material ( $\mathrm{Al} 4 \% \mathrm{Cu}$ ) and specimen geometry (Compact Tension) as in our own work. Figure 4 gives his results for a test conducted a load ratio, $R=0$. It can be seen that the measured $K_{F}$ value is very close to the nominal elastic $K$, calculated using the usual standard solution. The $K_{R}$ value starts close to zero, but then becomes negative, and increases in magnitude until the peak load, decreasing again during unloading.

If we now choose to plot a single crack driving force, equal to $K_{F}+K_{R}$, the results are transformed to those shown in Fig. 5. Finally, we note that in our own work, the datum for displacements was the 
unloaded specimen with the crack present, so that we are unable to detect any pre-existing residual K, and by definition the measured $\mathrm{K}$ must be zero at minimum load. However, Vasco-Olmo uses a different datum image and has a non-zero value of $\left(\mathrm{K}_{\mathrm{F}}+\mathrm{K}_{\mathrm{R}}\right)$ at the lowest applied load. Hence the appropriate parameter to plot in order to properly compare the two results is $\Delta\left(\mathrm{K}_{\mathrm{F}}+\mathrm{K}_{\mathrm{R}}\right)$. This parameter is given in Fig. 6, along with our own experimental data from Fig. 2, re-plotted on the same normalized axes. The comparison between the two sets of data, obtained independently on two different specimens in two different laboratories is striking, particularly when one considers that different DIC algorithms are used, and different post-processing routes were adopted.

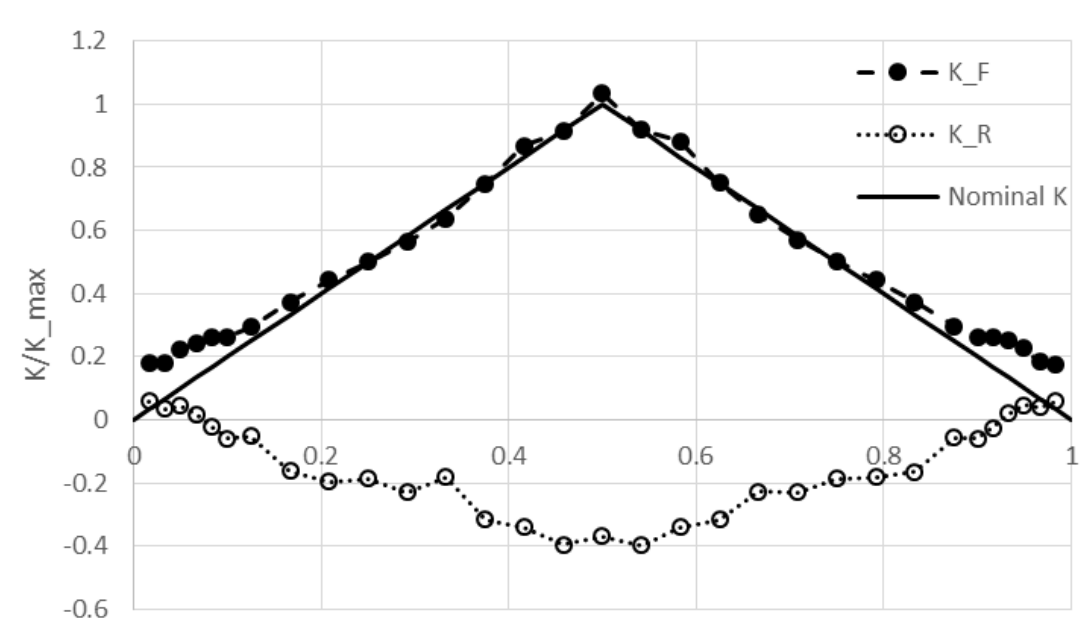

Normalised loading cycle

Figure 4: Results obtained by Vasco Olmo, [9], showing the variation of $K_{F}$ and $K_{R}$ through a load/unload cycle for an Al4\%Cu CT specimen. Results are normalized with respect to the nominal elastic K.

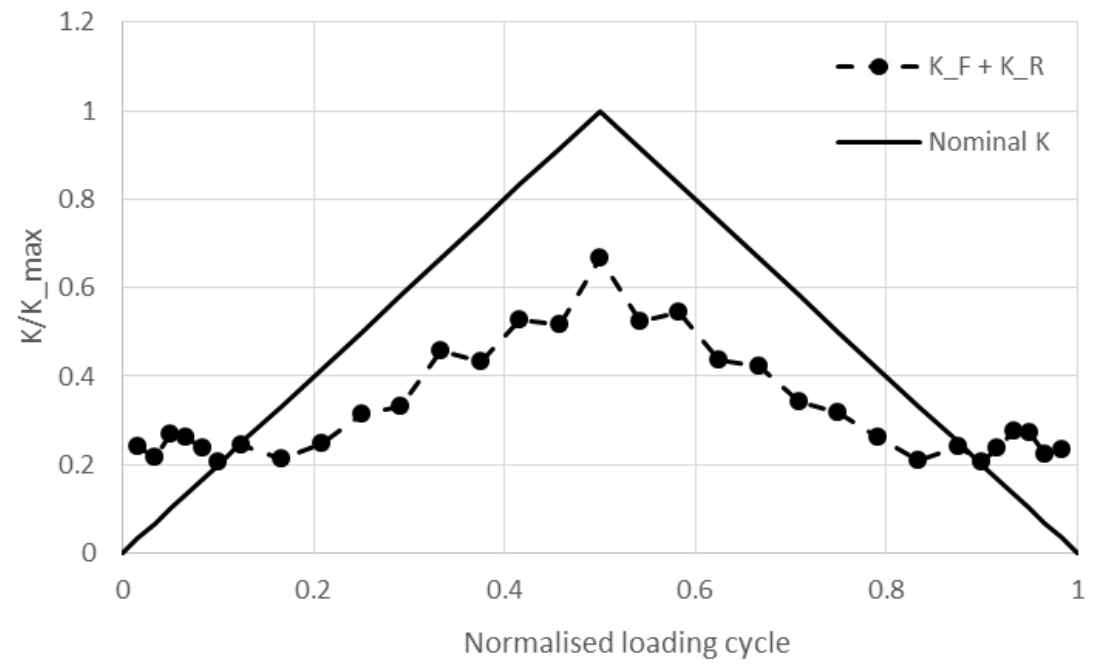

Figure 5: Results from Fig. 4, obtained by Vasco Olmo [9], re-plotted to show the variation of $\left(K_{F}+K_{R}\right)$ with loading cycle 
Comparison of these two sets of data, suggest that the four parameters of the mode I CJP model may usefully be reduced to three if one combines the $K_{F}$ and $K_{R}$ parameters, and that the three remaining terms each map clearly onto the effects of a force transmitted across the interface between the plastic zone and resulting wake, and the surrounding elastic hinterland. When viewed from this perspective, the combined $\mathrm{K}_{\mathrm{F}}+\mathrm{K}_{\mathrm{R}}$ parameter appears to be very similar to the measured delta $\mathrm{K}$ in our own experiments carried out under similar conditions. Hence, we are not yet convinced of the need to split $\Delta K$ in the way proposed by the CJP model. Nevertheless, we will proceed to show that a split of $\mathrm{K}$ into applied and residual terms may be helpful, even for measurements taken very close to the crack tip.

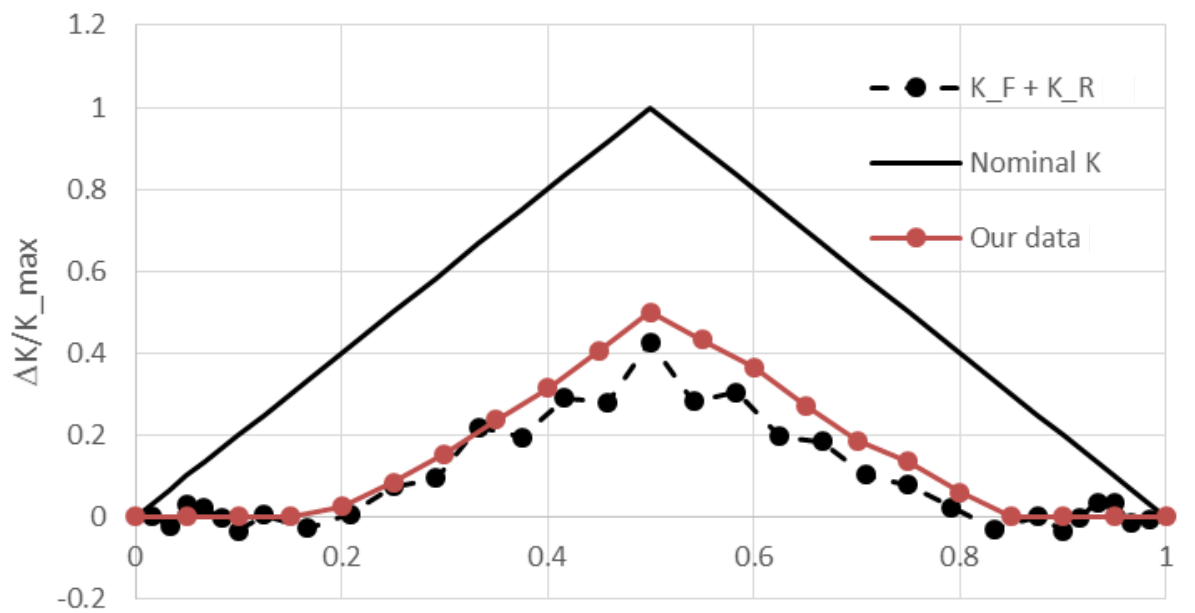

Normalised loading cycle

Figure 6: Vasco Olmo's data [9] plotted as delta K against loading cycle and compared against our own results[14]

\section{Micro-scale experiments}

The results obtained above suggest that a conventional elastic K-field may dominate the cyclic displacements measured around a growing fatigue crack, albeit with an offset due to crack closure, at least on the surface of a specimen or component. We will, of course, experience less closure away from the free surfaces, where the crack tip will be under plane-strain conditions. It is therefore interesting to examine the crack tip field in more detail, and we have therefore conducted a number of experiments using the same approach as above, but using a scanning electron microscope with and in-situ loading stage to image the crack tip at a high magnification. A similar DIC method may be used to analyse the images recorded, although one needs to be aware of particular issues which arise due to the scanned nature of the image [18]. A description of the experiment and some preliminary results has already been given in [19], but our intention here is to give a more in-depth treatment of the results obtained, particularly in the light of the discussion above. For clarity however, a brief summary of the experiment will first be presented. 


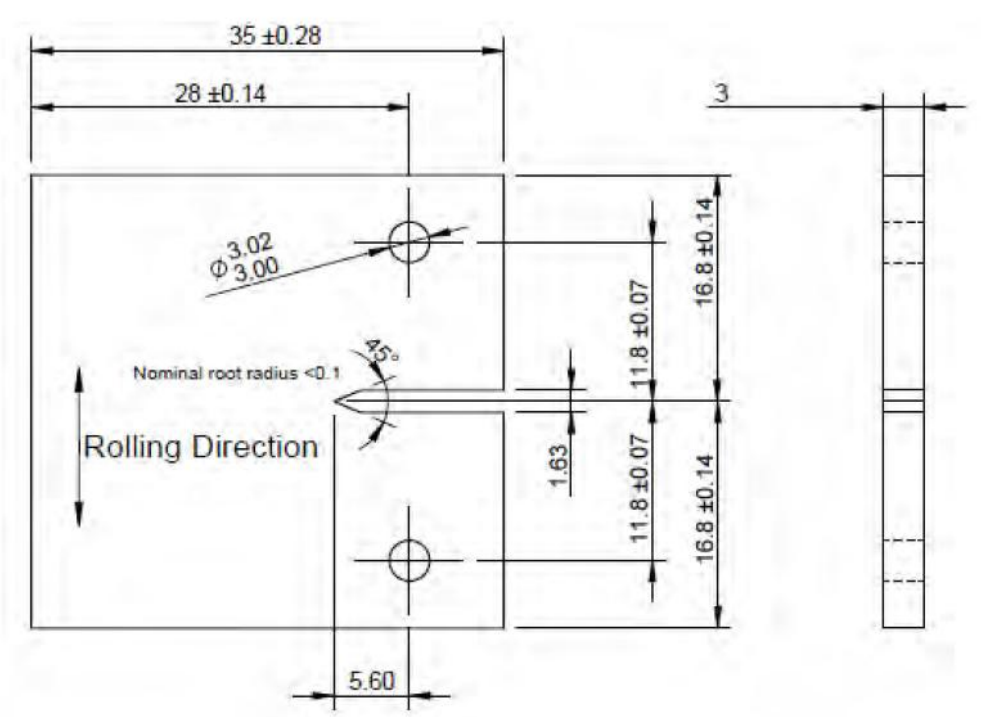

Figure 7: CT specimen used for in-situ testing in the Scanning Electron Microscope (dimensions in $\mathrm{mm}$ )

The imaging device used was a Carl Zeiss Evo LS15 VP-Scanning Electron Microscope and the chamber of the SEM was large enough so that in-situ testing could be performed with a $5 \mathrm{kN}$ Deben testing stage [19]. Specimens were made of Aluminium alloy 6082-T6, however the specimen size was reduced from the macroscopic tests in order to suit the loading stage are shown in Fig. 10. Specimens were pre-cracked on a conventional servo-hydraulic testing machine, before being tested in the SEM. After pre cracking, the specimens were unloaded, removed from the test machine and set-up on the Deben testing stage. Loading cycles were applied with a maximum force of $1.25 \mathrm{kN}$ and an $\mathrm{R}$ ratio of 0.1 . The time taken for a single cycle was approximately 140 seconds $(f=0.007 \mathrm{~Hz}$ ). In order to capture images with the SEM, the specimen loading needed to be paused during image capture. Hence, images could only be collected for relatively few cycles. Images were taken every $0.125 \mathrm{kN}$, giving 19 images for a complete cycle. After the image capture cycle, the load was increased to its maximum value and successive images were taken along the crack in order to determine the overall crack length when the crack was fully open.

Images were captured at a resolution of 3072 × 2304 pixels, giving an image area of approximately $215 \mu \mathrm{m} \times 161 \mu \mathrm{m}$ around the crack tip. As the loading applied to the specimen was adjusted between images, translational displacement of the crack tip occurred. Due to the high magnication of the images, the crack tip location was often displaced out of the previous image frame and therefore the testing stage position was adjusted within the SEM in order to place the crack tip in approximately the same position for all images. Images were later aligned using normalised 2-D cross-correlation with the MATLAB Image Processing Toolbox, in order to eliminate as much rigid body displacement as possible from the sequence of images. This preliminary processng was found to improve the performance of the subsequent digital image correlation. To analyse the captured images, the same MATLAB DIC code used in the previous experiments [19] was utilised with some minor adjustments. A grid of 2000 points was placed above and below the crack, Figure 8 . Each grid had 200 points in the $x$ direction (approximately parallel to the crack and 10 in the y direction normal to the crack. Due to the high resolution of the images, displacements around the crack were quite large at maximum load. In order to reliably determine the displacement of each point in the grid, a subset of $201 \times 201$ pixels was used to perform the correlation. This is a significant increase on the $31 \times 31$ pixel square that was 
used previously in the larger-scale experiments [19]. This increase signicantly increased DIC processing time.

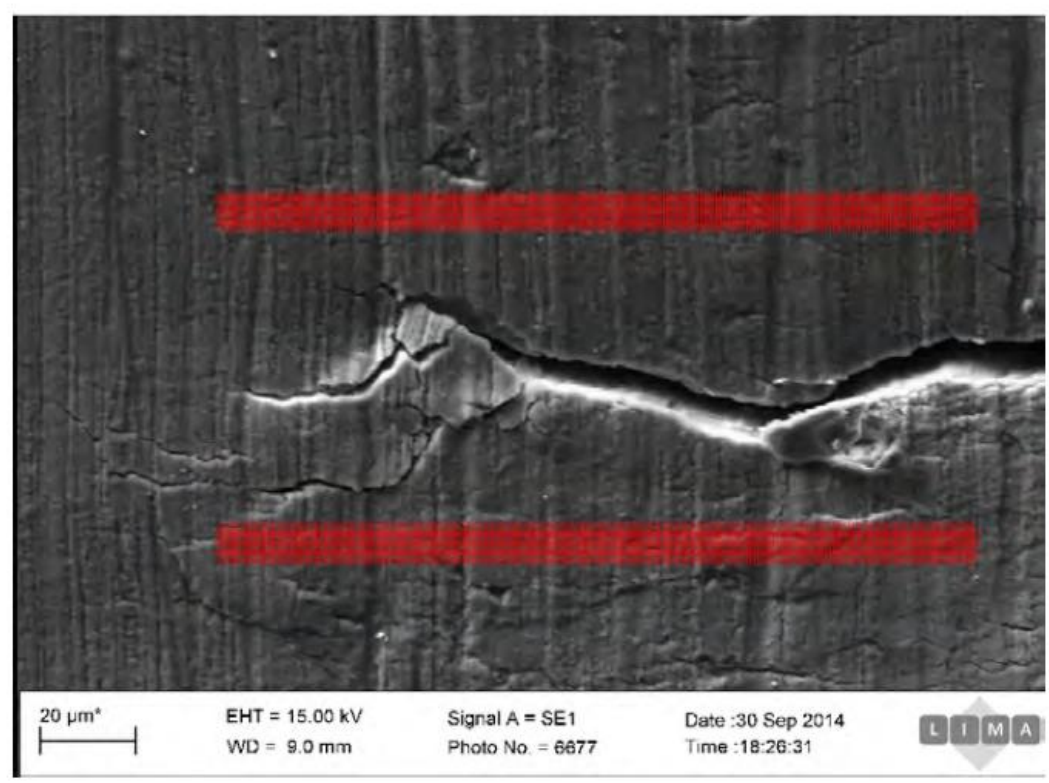

Figure 8: Typical image taken in the SEM (at maximum load), showing the grid of points used in the Digital Image Correlation. Note that it is fairly straightforward to determine the position of the main crack tip, even where there is some branching.

\subsection{Results}

The results reported here are for a specimen loaded with 17,760 cycles where the crack length was $7.18 \mathrm{~mm}$. Other cases were analysed, but all the constant amplitude tests gave essentially similar results. The displacement between sets of points in the two grids was the basis for analysis. A graph of the relative displacement between pairs of points for three distances from the crack tip are plotted against time (image number) and compared to normalised load $\left(P / P_{\max }\right)$ in Figure 9 . The differences between the loading and unloading phases of a cycle are noticeable in this plot and are more pronounced than in the macroscopic measurements [19]. In particular, the initial loading and initial unloading phases show displacements not responding at the same rate as they do during later stages of loading and unloading. This is more prominent at points very near the crack tip, which require a higher load before initial displacement and during initial unloading decrease in displacement more slowly than points further from the crack tip. 


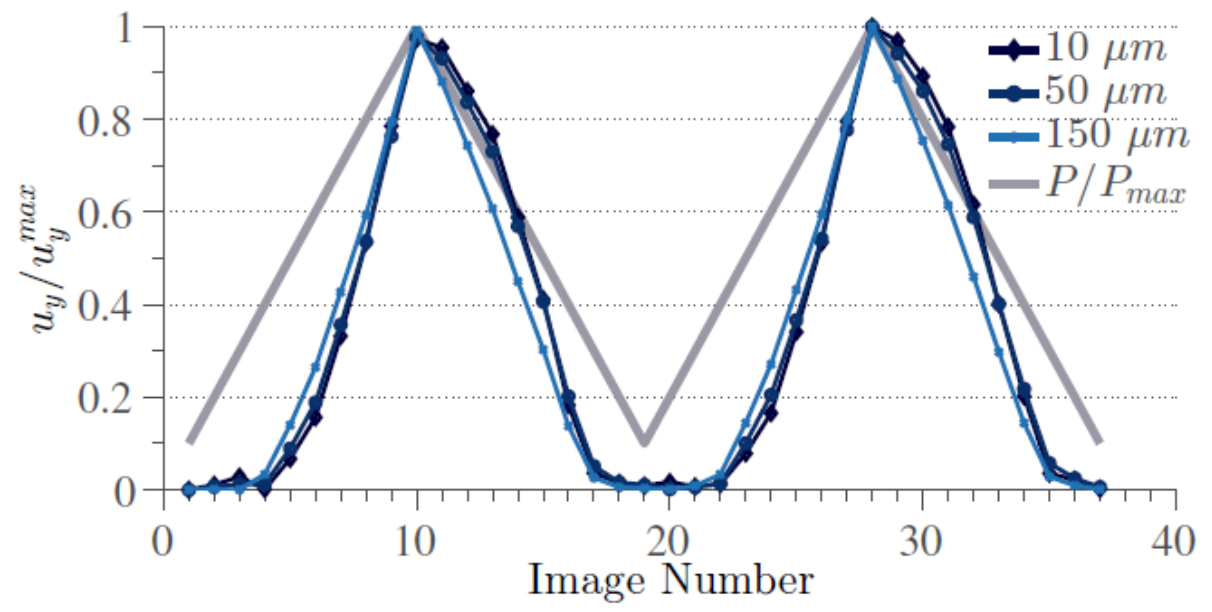

Figure 9: Typical variation of relative displacement $u_{y} / u_{y m a x} v s$ time (image number) compared to the applied load (P/Pmax). Relative displacement between pairs of points located at $10 \mu \mathrm{m}, 50 \mu \mathrm{m}$ and $150 \mu \mathrm{m}$ from the crack tip is shown.

The higher opening load for points near the crack tip is reflected in the corresponding plot of relative load vs displacement (Figure 10) when looking at points $10 \mu \mathrm{m}$ and $150 \mu \mathrm{m}$ from the crack tip. At points $10 \mu \mathrm{m}$ from the crack tip, the displacement between the points only begins to noticeably increase when $P / P_{\max }>0: 4$, while at $150 \mu \mathrm{m}$ the required load is only $P / P_{\max }>0: 3$. This is consistent with previous experiments and in this case improved resolution of opening load is provided by using points close to the crack tip.

Once again, the displacements of points along the crack flanks can be compared to those calculated from the elastic solution for a crack in the same specimen geometry. Figure 10 shows the displacements at various points in a loading cycle during both loading and unloading. In all cases, the experimentally obtained displacement values are significantly lower than those calculated. This is different from the macroscopic experiment, where the calculated displacement agreed well with that measured on the specimen. The main reason for the difference is the relatively high crack opening load that has a greater effect on points near the crack tip than those further along the crack flanks. In Figure $10 f(P=0.5 \mathrm{kN}, P / P \max =0: 4)$, for example, there is very little displacement along the whole section of crack examined, while the calculated displacement reaches $u_{y}=4 \mu \mathrm{m}$ at $190 \mu \mathrm{m}$ from the crack tip. This offset between the calculated displacements and those observed stays consistently near this value at higher loads.

An elastic model can be used, as described previously, to analyse the crack tip displacements and obtain a value for $K$. The linear regression lines fitted to $u_{y} v s \sqrt{r}$ plots give good fits with $R^{2}$ values of $0: 935<R^{2}<0: 999$ for loads $P / P_{\max }>0: 4$, where the crack is fully open to the tip. 


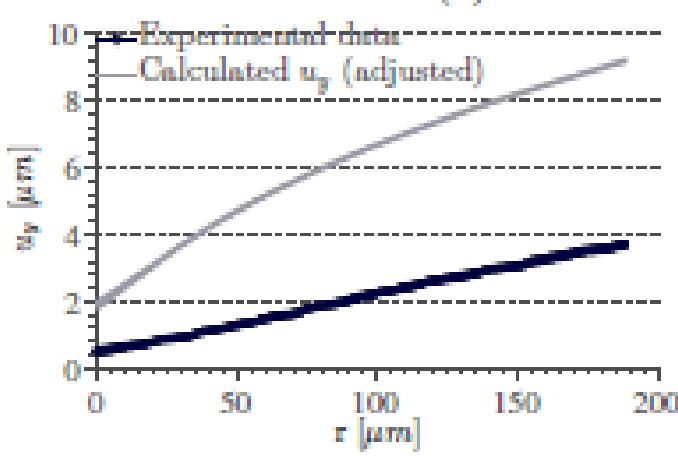

(b) $P=1.0 \mathrm{kN}$, Loading

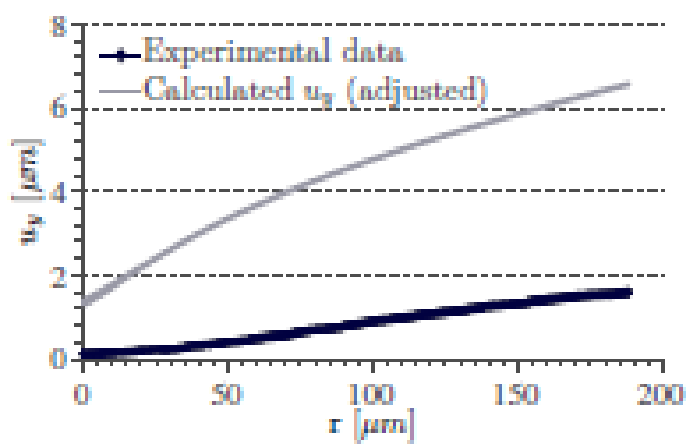

(d) $P=0.75 \mathrm{kN}$, Loading

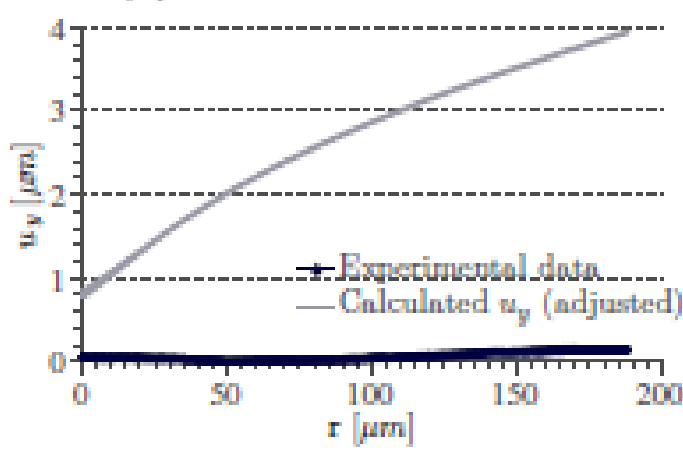

(f) $P=0.5 \mathrm{kN}$, Loading

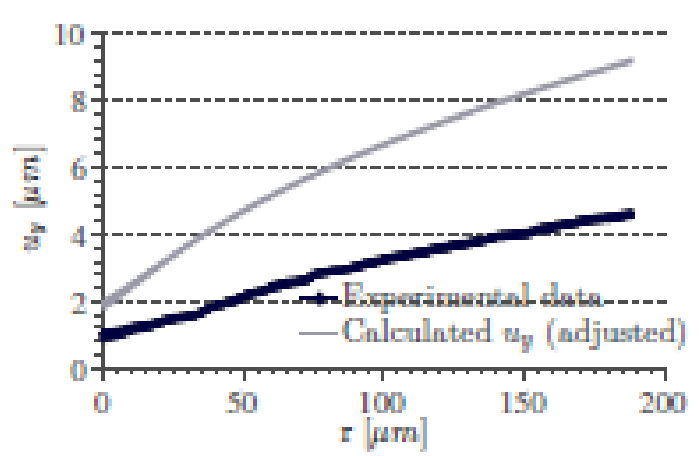

(c) $P=1.0 \mathrm{kN}$, Unloading

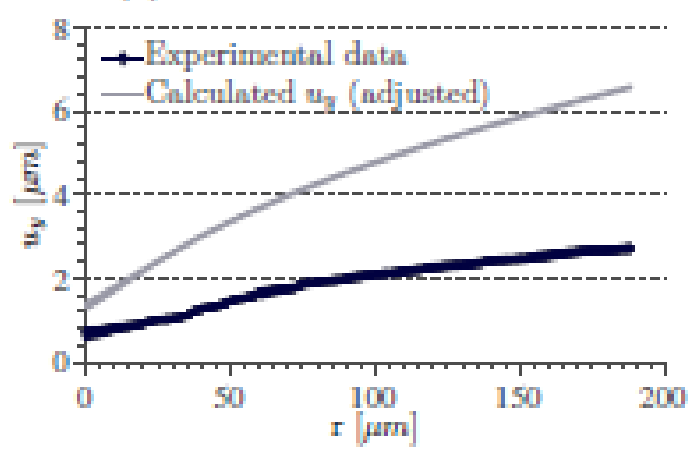

(e) $P=0.75 \mathrm{kN}$, Unloading

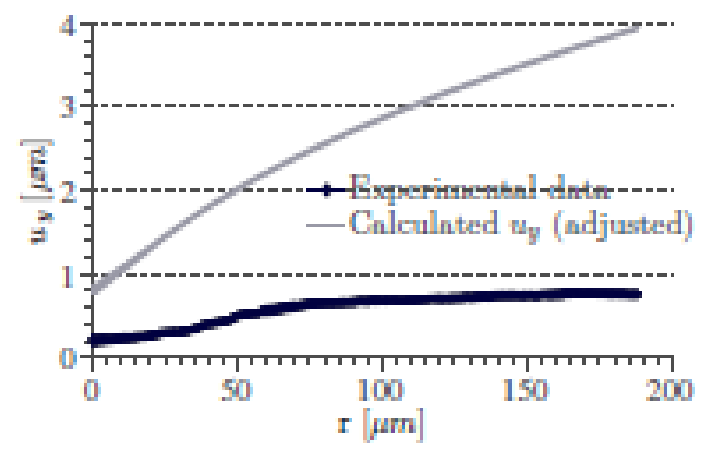

(g) $P=0.5 \mathrm{kN}$, Unloading

Figure 10: Measured displacement vs $r$ for various stages in a loading cycle. The displacement calculated from the elastic displacement field is plotted for comparison. 


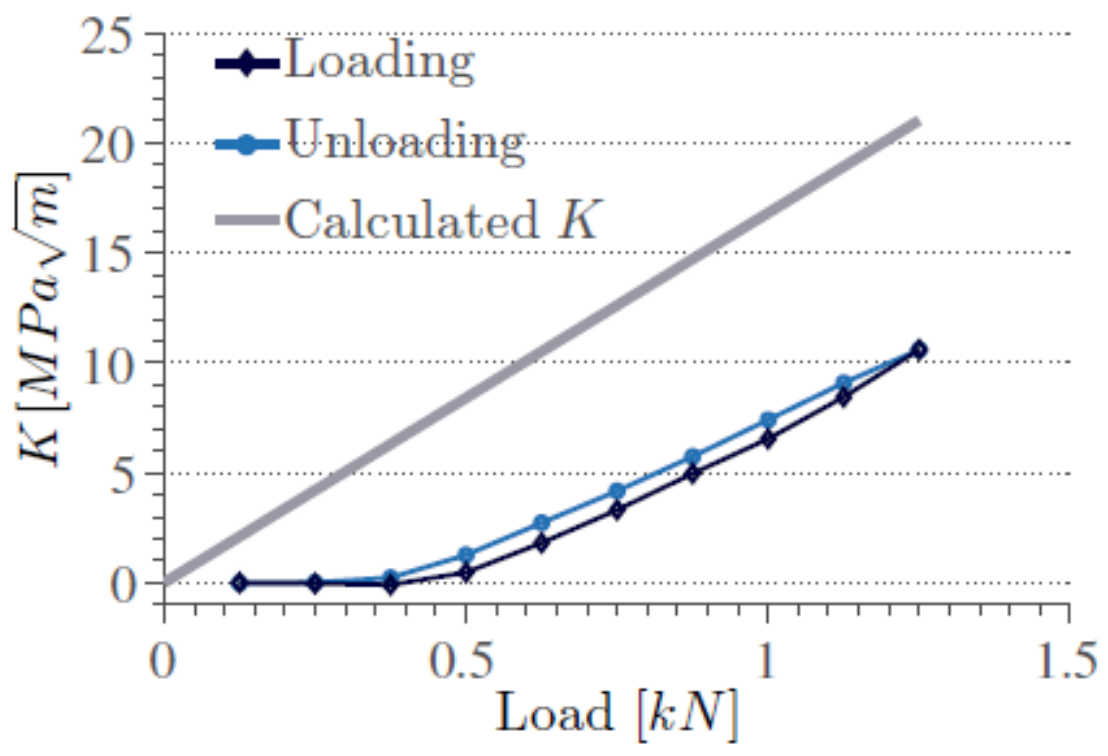

Figure 11. Variation of measured and calculated stress intensity factor with load, from analysis of the data shown in Figure 10.

\section{$\underline{\text { 4. Conclusions }}$}

The paper has described further results from in-situ loading of a crack tip in a scanning electron microscope. The feasibility of such measurements has been demonstrated and useful results have been obtained. A critical analysis has been undertaken of the CJP model for crack tip fields. It has been shown that the full four parameter description is not needed in order to give a good fit to the measured displacement. For the results described here, a two parameter description is sufficient: a conventional elastic stress intensity factor caused by the applied load, and a constant residual stress intensity factor caused by the residual stress field set up by crack tip and crack wake plasticity. At low loads, this residual stress intensity manifests itself as crack closure, and the boundary conditions of the problem are modified. It should be recognized that the results shown are purely surface measurements, and more data is required to fully understand the crack tip behaviour remote from the surface. In principle, this may be obtained by digital volume correlation of tomographic data, but the experiments are far more difficult than those described here. However, it would appear that, for the loads applied here at least, $\mathrm{K}$ is still a useful parameter in characterizing crack behaviour. Finally, we observe that many of our images show some signs of micro-cracks and crack tip branching in the plastic zone (see Figure 8 ), and this remains a phenomenon to be further investigated.

\section{$\underline{\text { 5. Acknowledgement }}$}

The authors gratefully acknowledge the help of Dr José Vasco Olmo, particularly in supplying the original data corresponding to figures 4, 5, and 6. Dr Vasco Olmo and Dr Paco Diaz are also thanked for helpful discussions during Professor Nowell's research visit to Jaén in May 2016, and both are further thanked for their kind and generous hospitality. 
[1] Paris, P., Erdogan, F. A critical analysis of crack propagation laws, J. Basic Eng, 85(1963), 528-34.

[2] Pommier, S., Hamam, R., Incremental model for fatigue crack growth based on a displacement partitioning hypothesis of mode I elastic-plastic displacement fields, Fatigue Fract. Engng Mater. Struct.,30(2006) 582-598.

[3] Christopher, C.J., James, M.N., Patterson, E.A., Tee, K.F., Towards a new model of crack tip stress fields. Int. Jnl Fracture, 148(2007), 361-371.

[4] Fellows, L.J., and Nowell, D., 'Crack closure measurements using moiré interferometry with photoresist gratings', Int. Jnl. Fatigue, 26, 1075-1082, 2004.

[5]Hild, F., Roux, S, Digital Image Correlation: from displacement measurement to determination of elastic properties - a review, J. Strain Analysis, 42(2006), 69-80.

[6] de Matos, P.F.P., and Nowell, D., 'Experimental and numerical investigation of thickness effects in plasticity-induced fatigue crack closure', Int. Jnl Fatigue, 31, 1795-1804, 2009.

[7] Yoneyama, S., Ogawa, T., and Kobayashi, Y., 'Evaluating mixed-mode stress intensity factors from fullfield displacement fields obtained by optical methods', Eng Fract. Mech., 74(9), 1399-1412, 2007.

[8] Roux, S., Rethore, J., and Hild, F., 'Digital image correlation and fracture: an advanced technique for estimating stress intensity factors of 2D and 3D cracks', Jnl Phys D: Appl. Phys, 42(21), Art. No. 214004, 2009.

[9] Vasco Olmo, J.M., Experimental evaluation of plasticity induced crack shielding effect using fullfield optical techniques for stress and strain measurement, PhD thesis, University of Jaén, 2014.

[10] Barhli, S.M., Mostafavi, M., Cinar, A.F., Hollis, D., and Marrow, T.J., 'J J-Integral Calculation by Finite Element Processing of Measured Full-Field Surface Displacements', Exp. Mech., 57(6), 997-1009, 2017.

[11] Maire, E., and Withers, P.J., 'Quantitative X-ray tomography', Int. Mats Reviews, 59(1) 2014.

[12] Limodin, N., Rethore, J., and Buffiere, J.-Y. 'Crack closure and stress intensity factor measurements in nodular graphite cast iron using three-dimensional correlation of laboratory X-ray microtomography images', Acta Mat., 57(14), Pages: 4090-4101, 2009.

[13] Lopez-Crespo, P., Steuwer, A., Buslaps, T., Tai, Y. H., Lopez-Moreno, A., J. R., and Withers, P. J., 'Measuring overload effects during fatigue crack growth in bainitic steel by synchrotron $\mathrm{X}$-ray diffraction', Int. Jnl Fatigue, 71, 11-16, 2015.

[14] Nowell, D., Kartal, M.E., de Matos, P.F.P., 'Measurement and modelling of near-tip displacement fields for fatigue ecracks in 6082 T6 aluminium', Proc. First I.J. Fatigue \& FFEMS Joint Workshop, Forni di Sopra, Italy, March 7-9,2011, Gruppo Italiano Frattura, 2011.

[15] Nowell, D., Kartal, M.E.,de Matos, P.F.P., 'Characterisation of crack tip fields under non-uniform fatigue loading', Proc. Second I.J. Fatigue \& FFEMS Joint Workshop, Malaga, Spain, April 15-17, 2013, Gruppo Italiano Frattura, 2013.

[16] Nowell, D., O'Connor, S.J., Dragnevski, K.I. , 'Measurement and analysis of fatigue crack deformation on the macro- and micro-scale', Proc. Third I.J. Fatigue \& FFEMS Joint Workshop, Urbino, Italy, April 20-22, 2015, Gruppo Italiano Frattura, 2015.

[17] Westergaard, H.M., "Bearing Pressures and Cracks," Journal of Applied Mechanics, 6(1939), A4953.

[18] Kammers, A.D., and Daly, S., 'Digital image correlation under scanning electron microscopy: methodology and validation', Exp. Mech., 53(2013), 1743-61.

[19] O'Connor, S.J., Nowell, D. and Dragnevski, K.I., 'Measurement of fatigue crack deformation on the macro- and micro-scale: uniform and non-uniform loading', Int Jnl Fatigue, 89, 66-76, 2016. 